\title{
Monoclonal immunoglobulin mediates complement activation in monoclonal gammopathy associated-C3 glomerulonephritis
}

Lin-Lin $\mathrm{Li}^{1,2+}$, Zhi-Ying Li ${ }^{1,2+}$, Su-Xia Wang ${ }^{3}$, Xiao-Juan $\mathrm{Yu}^{1,2}$, Ying Tan ${ }^{1,2}$, Yu Wang ${ }^{1,2^{*}}$, Feng $\mathrm{Yu}^{1,2,4^{*}}$ and Ming-Hui Zhao ${ }^{1,2,5}$

\begin{abstract}
Background: C3 glomerulonephritis (C3GN) is a rare disease caused by inherited or acquired complement alternative pathway (CAP) dysregulation, which could also be secondary to monoclonal gammopathy of undetermined significance (MGUS). Herein, we described a patient presenting with C3GN and monoclonal gammopathy, and the pathogenic association between the two diseases was further explored in vitro.

Case presentation: A 76-year-old Chinese man presented with low serum C3 level, haematuria and nephrotic syndrome, and experienced rapid worsening of renal function over a period of 10 months. His serum and urine immunofixation electrophoresis both revealed a monoclonal $\lg \mathrm{G} \lambda$. A bone marrow puncture showed plasma cell dyscrasias with the highest plasma cell count of 5.25\%. Kidney biopsy showed the presence of C3 glomerulonephritis, with exclusive deposits of C3 visible on immunofluorescence, a membranoproliferative pattern on light microscopy and electron dense deposits in sub-epithelial, intramembranous, sub-endothelial and mesangial regions by electron microscopy. The patient was positive for $\mathrm{C} 3$ nephritic factor (C3NeF) activity and anti-CFH autoantibodies, and all became negative during disease remission. The anti-CFH autoantibodies purified from the patient's plasma exchange fluids were proven to be a monoclonal IgG $\lambda$, and could inhibit CFH binding to C3b and accelerate the formation of C3 convertase indirectly by interfering with the formation-impeding activity of CFH. No deficiency of candidate genes, especially variants in CFH, was detected in our patient. Based on the pathological and laboratory findings, the diagnosis of monoclonal gammopathy of renal significance (MGRS)-associated C3GN was finally made.
\end{abstract}

Conclusions: This is the first demonstration that intact monoclonal immunoglobulin (IgG $\lambda$ ) could act as an anti-CFH antibody and lead to MGRS-associated C3GN by activating the CAP.

Keywords: C3 glomerulonephritis, Anti-CFH autoantibodies, Monoclonal immunoglobulin (Mlg), Monoclonal gammopathy of renal significance (MGRS)

\footnotetext{
* Correspondence: ddwangyu@sina.com; yufengevert1@sina.com

${ }^{\dagger} \mathrm{Lin}-\mathrm{Lin} \mathrm{Li}$ and Zhi-Ying Li contributed equally to this work.

${ }^{1}$ Renal Division, Department of Medicine, Peking University First Hospital,

Institute of Nephrology, Peking University, Beijing 100034, People's Republic

of China

Full list of author information is available at the end of the article
}

(c) The Author(s). 2019 Open Access This article is distributed under the terms of the Creative Commons Attribution 4.0 International License (http://creativecommons.org/licenses/by/4.0/), which permits unrestricted use, distribution, and reproduction in any medium, provided you give appropriate credit to the original author(s) and the source, provide a link to the Creative Commons license, and indicate if changes were made. The Creative Commons Public Domain Dedication waiver (http://creativecommons.org/publicdomain/zero/1.0/) applies to the data made available in this article, unless otherwise stated. 


\section{Background}

C3 glomerulopathy (C3G) is characterized by predominant glomerular C3 fragment deposition with electrondense deposits on electron microscopy. The disease is thought to be caused by excessive activation of the complement alternative pathway (CAP) and serum C3 levels are usually low. According to the distribution of electron-dense deposits on electron microscopy, C3G could be subdivided into dense deposit disease (DDD) and $\mathrm{C} 3$ glomerulonephritis (C3GN) [1, 2]. C3G results from acquired or genetic abnormalities in the CAP, such as the presence of $\mathrm{C} 3$ nephritic factor $(\mathrm{C} 3 \mathrm{NeF})$, antibodies or gene variants/mutations for complement factor $\mathrm{H}(\mathrm{CFH})$ or complement factor B (CFB), etc. [3, 4]. Monoclonal immunoglobulins ( $\mathrm{MIg}$ ) may also play a causal role in C3G by impairing the regulation of the CAP [5]. The terminology MGRS (monoclonal gammopathy of renal significance) is used to denote a monoclonal gammopathy of undetermined significance that is responsible for a renal disease [6, 7]. Recently, a link between C3G, monoclonal gammopathy and MGRS has been observed, especially in older adults [8-15], although the role of MIg in the pathogenesis of C3G remains to be elucidated. We herein described a patient presenting with $\mathrm{C} 3 \mathrm{GN}$ and monoclonal gammopathy, and the pathogenic association between the two diseases was further explored in vitro.

\section{Case presentation \\ Case descriptions}

A 76-year-old Chinese man presented with microscopic haematuria for 2 years and oedema for 8 months. The patient had a past history of age-related macular degeneration (AMD), hypertension, angina pectoris and hypothyroidism. On admission, the physical examination revealed a blood pressure of $145 / 76 \mathrm{mmHg}$, a temperature of $36.5{ }^{\circ} \mathrm{C}$, a heart rate of 76 beats $/ \mathrm{min}$, and a respiratory rate of 22 breaths $/ \mathrm{min}$. The patient had severe bilateral symmetrical lower extremity oedema. Urine dipstick showed blood (3+) and protein $(3+)$, and urinalysis showed with 80-90 RBCs/HPF with a majority of dysmorphic RBCs. Laboratory findings included a serum albumin concentration of $20 \mathrm{~g} / \mathrm{L}$ and a proteinuria value of $8.06 \mathrm{~g} / \mathrm{d}$. His serum creatinine value rose from $1.41 \mathrm{mg} / \mathrm{dL}$ to $2.96 \mathrm{mg} / \mathrm{dL}$ in 10 months and decreased to $1.81 \mathrm{mg} / \mathrm{dL}$ after diuretic therapy. His
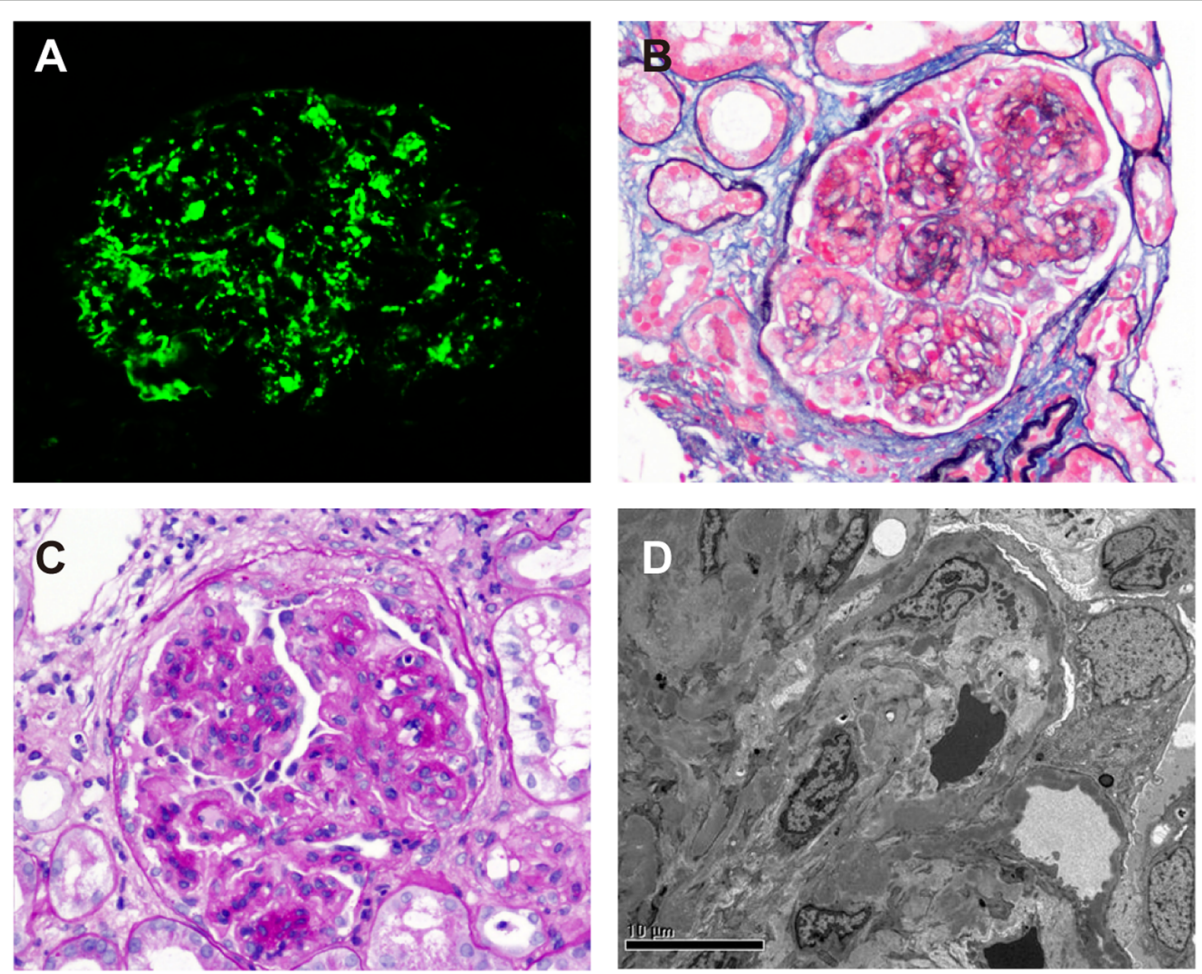

Fig. 1 Representative figures of renal biopsy. a Intensive granular deposits of C3 $(3+-4+)$ were detected in the mesangial regions and segmental deposits along the capillary walls (immunofluorescence staining on frozen tissue, $\times 400$ ). b Severe mesangial proliferation and interposition to form a lobular appearance, along with a thickening of the glomerular capillary wall with double contours (periodic acid-silver methenamine + Masson trichrome staining, $\times 400)$. c Severe mesangial proliferation with endocapillary hypercellularity and a small fibro-cellular crescent (Periodic acid staining, $\times 400$ ). $\mathbf{d}$ Electron dense deposits in sub-endothelia, intramembranous and mesangial regions viewed by electron microscopy $(\times 8000)$ 
haemoglobin level was $77 \mathrm{~g} / \mathrm{L}$ (normal range: $130-175 \mathrm{~g} / \mathrm{L}$ ) and his platelet count was $212 \times 109$ cells/L (normal range: $125-350 \times 109$ cells $/ \mathrm{L}$ ). His C3 level was low at $0.356 \mathrm{~g} / \mathrm{L}$ (normal range: $0.6-1.5 \mathrm{~g} / \mathrm{L}$ ), his $\mathrm{C} 4$ level was normal at $0.162 \mathrm{~g} / \mathrm{L}$ (normal range: $0.12-0.36 \mathrm{~g} / \mathrm{L}$ ) and his plasma $\mathrm{CFH}$ level was normal at $392.9 \mu \mathrm{g} / \mathrm{mL}$ (normal range: $247-$ $1010.8 \mu \mathrm{g} / \mathrm{mL}$ ). His serum IgG level was $9.89 \mathrm{~g} / \mathrm{L}$ (normal range: $7.23-16.85 \mathrm{~g} / \mathrm{L}$ ), his IgA level was $2.38 \mathrm{~g} / \mathrm{L}$ (normal range: $0.69-3.82 \mathrm{~g} / \mathrm{L}$ ), and his IgM level was $0.78 \mathrm{~g} / \mathrm{L}$ (normal range: $0.63-2.77 \mathrm{~g} / \mathrm{L}$ ). His serum and urine immunofixation electrophoresis both revealed a monoclonal IgG $\lambda$. A bone marrow puncture was performed, and the diagnosis of plasma cell dyscrasias was made, with the highest plasma cell count of $5.25 \%$.

The patient underwent a renal biopsy 2 days after hospitalization. By immunofluorescence, intensive granular deposits of $\mathrm{C} 3(3+-4+)$ were detected in the mesangial regions and segmental deposits along the capillary walls (Fig. 1a). C1q was trace, and no deposits of IgG, IgA, IgM, or $\mathrm{K}$ and $\lambda$ light chains were detected. By light microscopy, there were 12 glomeruli in the specimen, and the appearance of glomerular lesions was characterized by severe mesangial proliferation and interposition and endocapillary hypercellularity to form lobular and thickened glomerular basement membranes (GBMs) with double contours. Fuchsinophilic deposits were identified in the sub-endothelial and mesangial regions. Two of 12 glomeruli showed small fibro-cellular crescents. Degenerative changes in tubular epithelia were mild and a focal interstitial infiltration of lymphocytes with fibrosis was identified. Arteriolar sclerosis was mild (Fig. 1b and c). Electron microscopy revealed moderate to severe mesangial proliferation with interposition and a thickening of the GBM with a segmental widening of sub-endothelia regions was found. Electron-dense deposits were present in the sub-epithelial, intramembranous, sub-endothelial and mesangial regions (Fig. 1d). A severe foot process effacement of podocytes was identified. A final diagnosis of $\mathrm{C} 3$ glomerulonephritis with a pattern of membranoproliferative glomerulonephritis (MPGN) was then made.

\section{Detection of plasma C3NeF activity and anti-CFH or CFH SCR autoantibodies}

The patient was positive for $\mathrm{C} 3 \mathrm{NeF}$ activity. His anti-full length $\mathrm{CFH}$ and $\mathrm{CFH}$ SCR7 autoantibodies were also

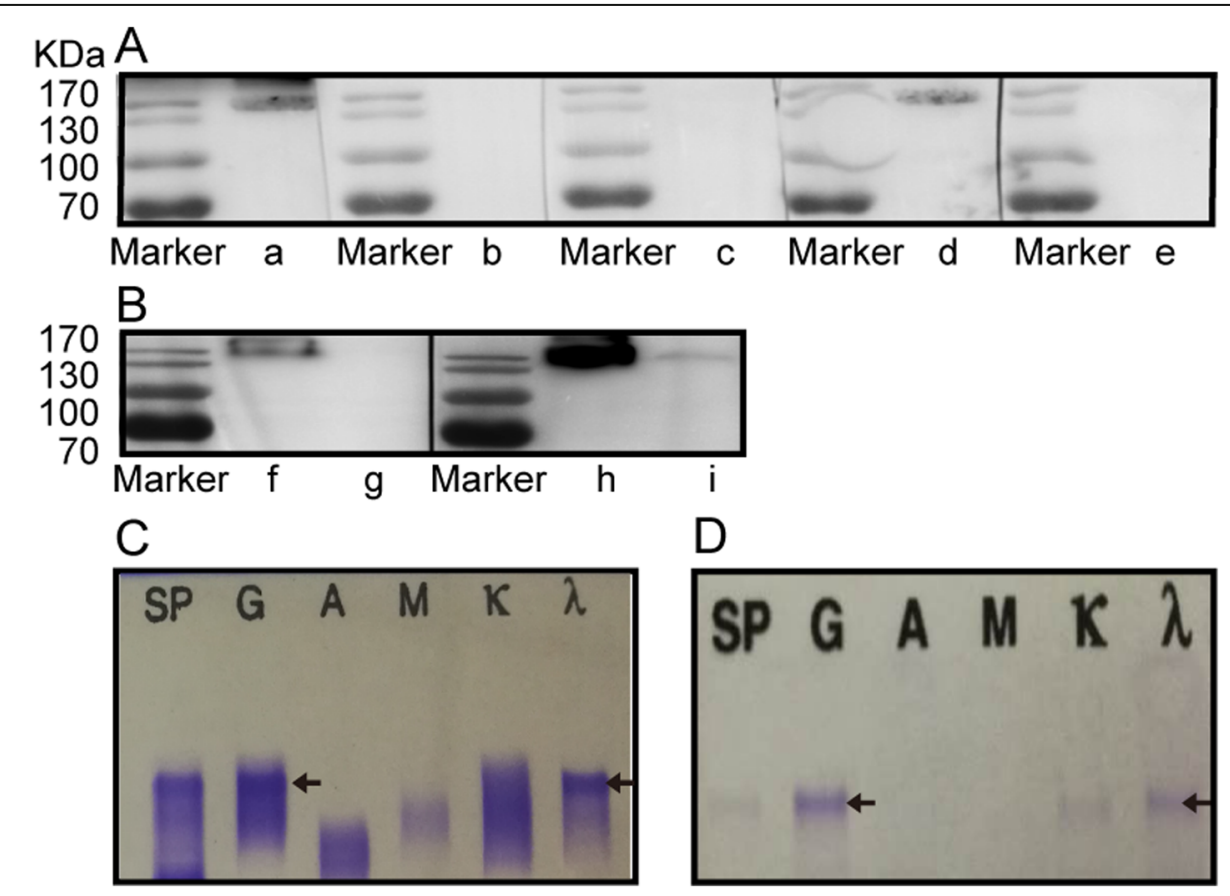

Fig. 2 The purified anti-CFH autoantibody. a Confirmation of purified anti-CFH autoantibodies from our patient by Western blot. Commercial human CFH $(1 \mu \mathrm{g})$ under non-reducing conditions was electrophoresed on 10\% SDS-PAGE and transferred to a PVDF paper, blocked and then incubated with anti-CFH autoantibodies purified from our patient. The anti-CFH autoantibodies purified from our patient, which was IgG3 $\lambda$, could recognize commercial CFH. Lanes a-e were incubated with the followings antibodies: anti-human $\operatorname{lgG}, \operatorname{lgG} 1$, $\operatorname{lgG} 2$, lgG3, and lgG4, respectively. b The anti-CFH autoantibodies purified from our patient had lambda light chain without a kappa chain. Lanes $f$ and $h$, lanes $g$ and $i$ were loaded with anti-CFH autoantibodies purified from the aHUS patient and our patient, respectively, under non-reducing conditions on 8\% SDS-PAGE; lanes $f$ and $g$ were then incubated with anti-human kappa chain, and lanes $h$ and $i$ were incubated with anti-human lambda chain. c The serum of our patient contained a monoclonal $\lg \mathrm{G} \lambda$ by immunofixation electrophoresis assay. $\mathbf{d}$ The purified anti-CFH autoantibodies from our patient were confirmed to be the same monoclonal $\lg \mathrm{G} \lambda$ by immunofixation electrophoresis assay 
found to be positive by ELISA, and the subclass of antifull-length CFH autoantibodies was IgG3.

His anti-CFH autoantibodies were purified from plasma exchange fluids using Protein G and CFH affinity column, and were proven to be a monoclonal IgG $\lambda$ by both Western blot and immunofixation electrophoresis assays, which was consistent with the serum monoclonal IgG入 (Fig. 2a-d).

\section{Bio-functional analyses of anti-CFH autoantibodies} in vitro

\section{C3b binding assay}

As shown in Fig. 3, the binding of $\mathrm{CFH}$ to $\mathrm{C} 3 \mathrm{~b}$ was reduced significantly in a dose-dependent manner with autoantibodies from our patient compared with IgG from 3 healthy controls $(P<0.001)$, which was even worse than the level interfered by the anti-CFH autoantibodies from an aHUS patient $(P=0.001)$.

\section{C3 convertase formation and decay assay}

We tested the capacity of CFH to interfere with the formation and accelerate the decay of the alternative pathway $\mathrm{C} 3$ convertase $(\mathrm{C} 3 \mathrm{bBb})$, which was assembled on immobilized C3b. Commercial CFH exhibited strong formation-impeding and decay-accelerating activity in a dose-dependent manner (Fig. 4a and b). Auto-antibodies against $\mathrm{CFH}$ purified from our patient showed significantly reduced formation-impeding activities in a dosedependent manner compared with IgG from 3 healthy controls and the aHUS patient (Fig. 4c). There was no significant difference in the decay-accelerating activities between any purified antibodies with commercial $\mathrm{CFH}$ and commercial $\mathrm{CFH}$ only for the $\mathrm{C} 3$ convertase decay (Fig. 4d).

\section{Genotyping}

An integrated screening of 34 genes from the complement system using targeted genomic enrichment and massively parallel sequencing was performed, including C1QA, C1QB, C1QC, C1R, C1S, C2, C3, C5, C6, C7, C8A, C8G and C9; Plasma protease $\mathrm{C} 1$ inhibitor (SERPING1); Complement factor B, D, P, I and $\mathrm{H}$; Complement factor $\mathrm{H}$ related protein 1-5; Thrombomodulin (THBD); Mannosebinding lectin serine protease 1 and 2 (MASP1, MASP2); Collectin-11 (COLEC11); Complement receptor type 2 (CR2); Integrin beta-29 (ITGB2); Membrane cofactor protein 46 (CD46); Membrane cofactor protein 59 (CD59); and Ficolin-3 (FCN3). No deficiency of a single gene or candidate genes, especially variants in $\mathrm{CFH}$, was detected in our patient.

\section{Final diagnosis, treatment and follow-up}

Based on the pathological and laboratory findings, the diagnosis of C3GN in our patient, which might be due to MGRS, was finally made (Detailed flow chart in Fig. 5). During the exploration of the underlying causes for C3GN in this patient, we found that the patient was

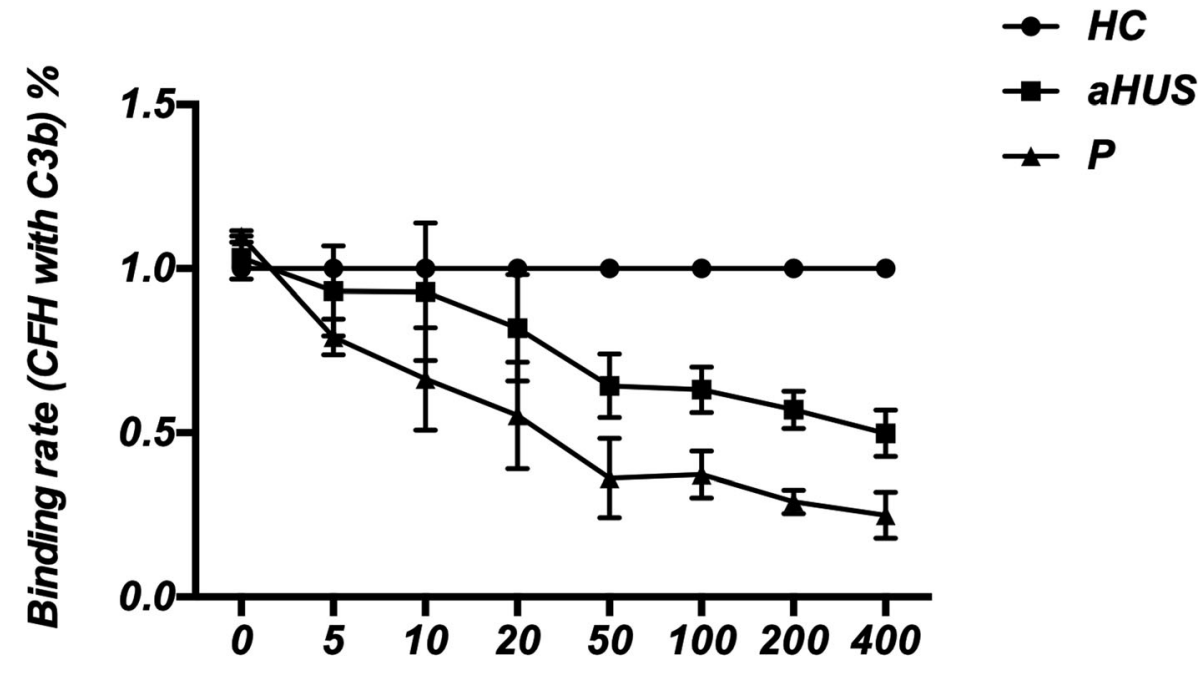

\section{Concentrations of anti-CFH autoantibodies/total lgG ( $\mu \mathrm{g} / \mathrm{ml})$}

Fig. 3 Binding assay of CFH to C3b. Inhibition of the interaction between CFH and C3b as measured by ELISA. The anti-CFH autoantibodies purified from our patient and the aHUS patient, and the total IgG from three healthy controls were analysed individually. The percentage of inhibition was calculated by defining the average optical density (OD) value obtained in the presence of each healthy control lgG to be 1 . The purified anti-CFH autoantibodies from our patient inhibited the interaction between CFH and C3b in a dose-dependent manner. Each assay was performed three times, and the data are shown as the mean \pm SD. Notes: P: Our patient; aHUS: the aHUS patient with anti-CFH autoantibodies; $\mathrm{HC}$ : healthy controls 


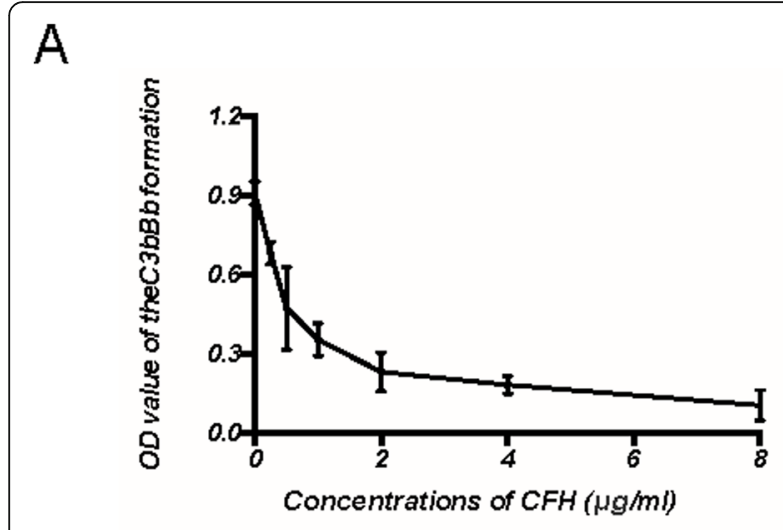

B

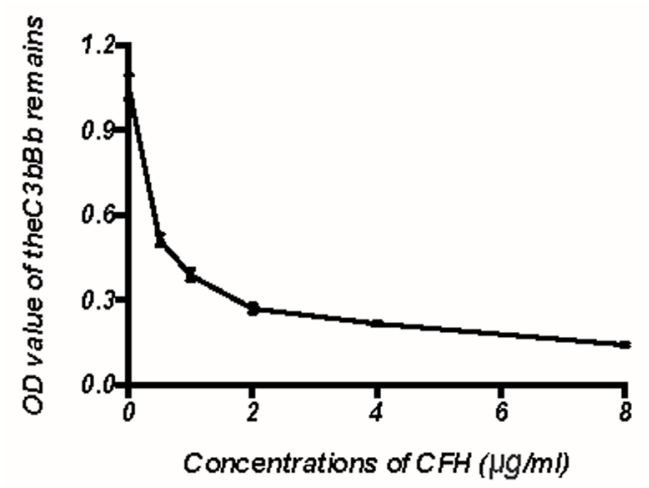

C

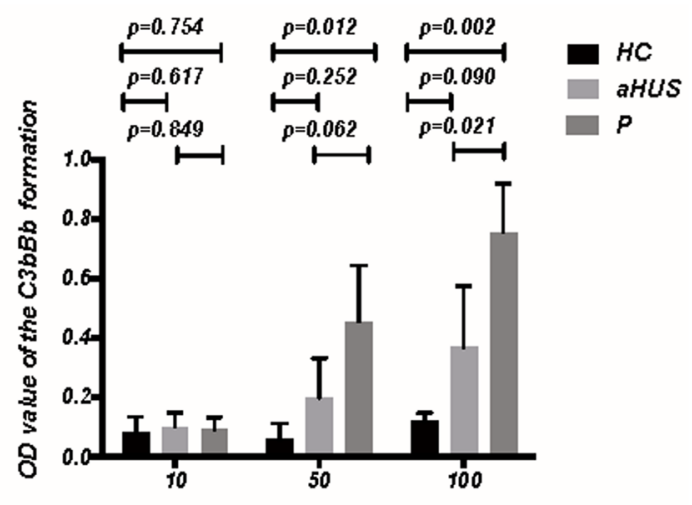

Concentrations of anti-CFH autoantibodies/totallgG $(\mathrm{kg} / \mathrm{ml})$

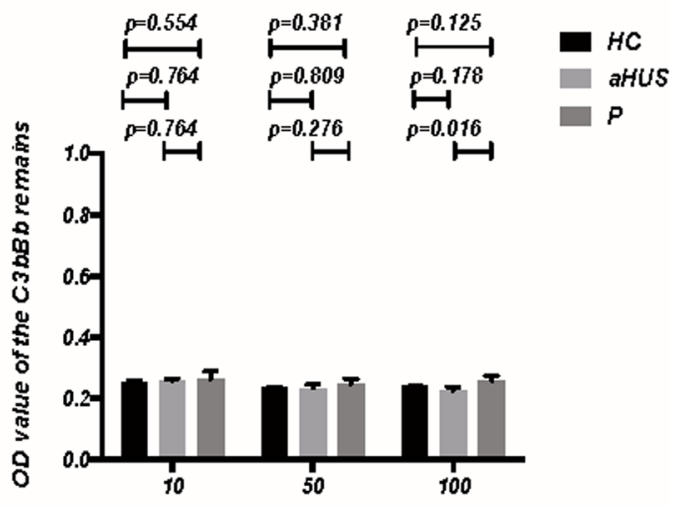

Concentrations of anti-CFH autoamtibodies/zotaligG $(\mu \mathrm{g} / \mathrm{m})$

Fig. 4 C3 convertase formation assay and decay assay. a Commercial human CFH exhibits a strong formation-impeding activity of the C3 convertase (C3bBb) assembled on immobilized C3b in a dose-dependent manner. $\mathbf{b}$ Commercial human CFH accelerates the decay of the C3 convertase (C3bBb) assembled on immobilized C3b in a dose-dependent manner. c, d Pre-incubated CFH (4 $\mu \mathrm{g} / \mathrm{ml})$ with purified anti-CFH autoantibodies from our patient and the aHUS patient, and total lgG from three healthy control individually at concentrations of $10 \mu \mathrm{g} / \mathrm{ml}, 50 \mu \mathrm{g} /$ $\mathrm{ml}$, and $100 \mu \mathrm{g} / \mathrm{ml}$, respectively. Anti-CFH autoantibodies purified from our patient showed significantly reduced formation-impeding activities in a dose-dependent manner (c) and no significant effects on the decay-accelerating activities of CFH (d). Notes: P: Our patient; aHUS: the aHUS patient with anti-CFH autoantibodies; HC: healthy controls. Each assay was performed three times, and the data are shown as the mean \pm SD

positive for $\mathrm{C} 3 \mathrm{NeF}$ activity and anti-CFH autoantibodies. His anti-CFH autoantibodies were proven to be a monoclonal $\operatorname{IgG} \lambda$, which corresponded to his serum and urine immunofixation electrophoresis results.

Six rounds of plasmapheresis were initiated, and the chemotherapy with bortezomib was then recommended although the patient declined the latter therapy. He received oral prednisone (initial daily dose of $1 \mathrm{mg} / \mathrm{kg}$, tapered slowly) and cyclophosphamide (daily dose of 50 $\mathrm{mg}$ ) with an accumulate doses of $4.5 \mathrm{~g}$ in combination with a calcium channel blocker and erythropoiesisstimulating agent therapy. Ten months later, his proteinuria amount had decreased to $0.24 \mathrm{~g} / \mathrm{d}$, his serum albumin level had increased to $40 \mathrm{~g} / \mathrm{L}$, his serum creatinine value was stable $(1.39-1.46 \mathrm{mg} / \mathrm{dL})$, his serum $\mathrm{C} 3$ level had increased to $0.92 \mathrm{~g} / \mathrm{L}$, his plasma $\mathrm{CFH}$ level had increased to $833 \mu \mathrm{g} / \mathrm{ml}$, and his plasma autoantibodies against CFH and plasma $\mathrm{C} 3 \mathrm{NeF}$ activity had both turned negative. His serum monoclonal $\lambda$ light chains were trace, and neither monoclonal IgG $\lambda$ nor monoclonal $\lambda$ light chains could be detected in his urine.

\section{Discussion and conclusions}

In this study, the definitive diagnosis of C3G was made based on the demonstration of $\mathrm{C} 3$ deposits by immunofluorescence and membranoproliferative glomerulonephritis (MPGN) and electron dense deposits in sub-epithelial, intramembranous, sub-endothelial and mesangial regions by electron microscopy [7] and monoclonal gammopathy was shown by the demonstration of a serum monoclonal IgG $\lambda$ by immunofixation electrophoresis assays. The associations between C3G and monoclonal gammopathy were further explored in vitro. 


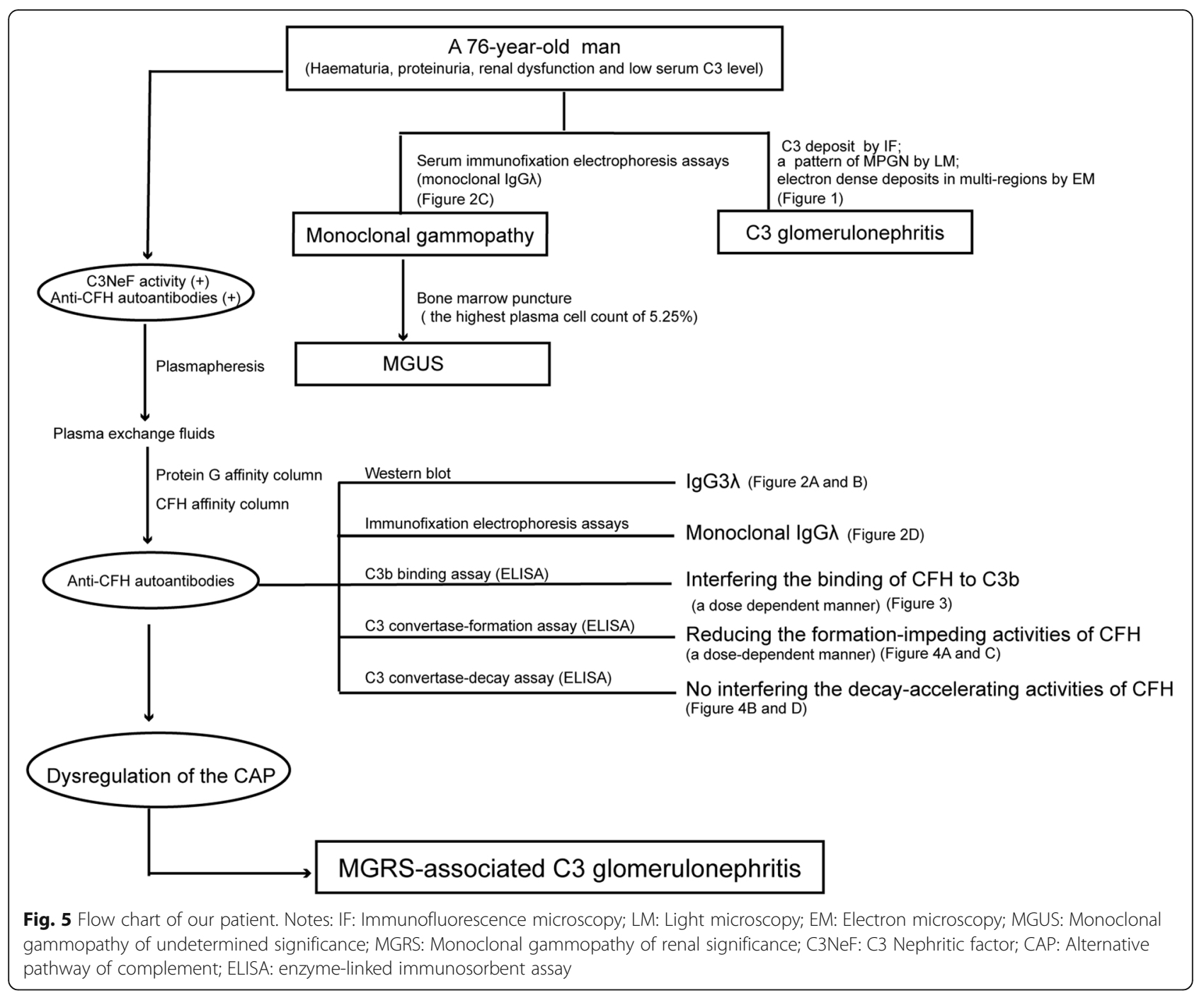

The mechanisms that contribute to C3G are multiple, including the presence of $\mathrm{C} 3 \mathrm{NeF}$, antibodies and/or gene variants/mutations of complement-regulating proteins, etc. [3, 4]. Recently, more attention was paid to the link between C3G and monoclonal gammopathy, especially in older adults $[5,8-10,12,13,16,17]$, as $65.1 \%$ of C3G patients with 50 years of age and older had a monoclonal gammopathy [16], which is nearly 16 times higher than the prevalence $(3.2 \%)$ of monoclonal gammopathy in the general population [18].

We reviewed and summarized published C3G combined monoclonal gammopathy cases in the Table 1 [5, 8-10, 12-17, 19-25]. In brief, C3G with monoclonal gammopathy mainly concerned older people, with a striking male predominance. IgG $\mathrm{k}$ was the most common MIg isotype, and the vast majority of the patients were classified as MGRS/MGUS. In renal ultrastructural pattern, C3GN was more common than DDD, although there was an overlap of cohort between Chauvet's two series studies. The renal outcomes of C3G was poor in most cases, and the improvement of renal parameters after chemotherapy in a few patients suggested that MIg might have a pathogenic role in CAP activation. In some cases, C3NeF, anti-CFH autoantibodies, and anti-CR1 autoantibodies could be detected, and it was assumed that MIg could act as an antibody to complement fragments such as $\mathrm{C} 3$ convertase or $\mathrm{CFH}$, interfering with bio-functions and resulting in the over-activity of the CAP $[8,9]$, although the precise mechanism remained to be elucidated. Importantly, we found that the subclass of anti-CFH autoantibodies in our patient was IgG3, which is poorly represented in normal polyclonal IgG. IgG is divided into four subclasses, named, in order of decreasing abundance IgG1, IgG2, IgG3 and IgG4, which are highly homologous and differ in their constant region, particularly in their hinges and upper $\mathrm{CH} 2$ domains [26]. These regions are involved in binding to both IgG-Fc receptors $(\mathrm{Fc} \gamma \mathrm{R})$ and C1q. IgG3 has a much longer region than any of the other 
Li et al. BMC Nephrology

(2019) 20:459

Page 7 of 10

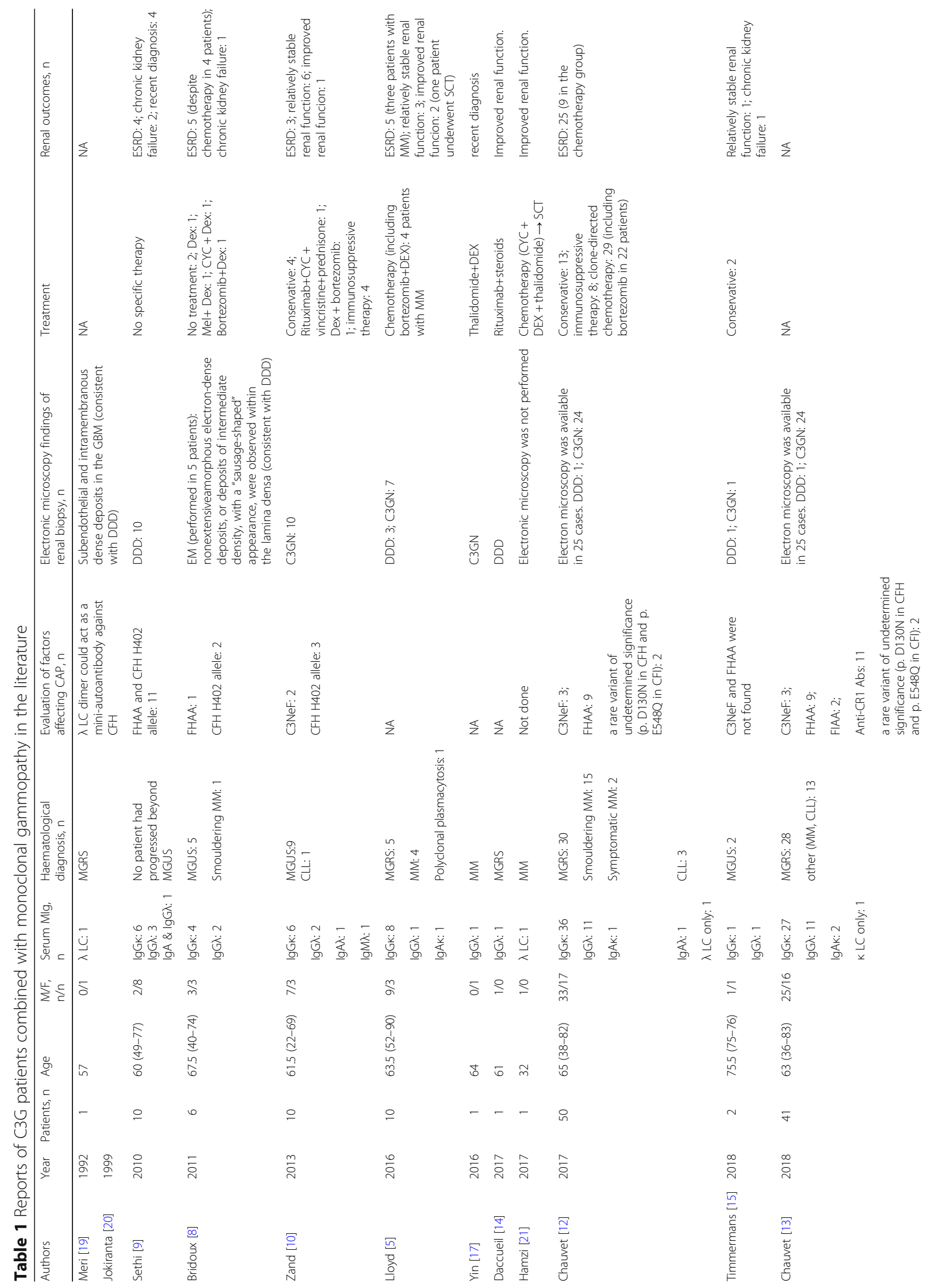




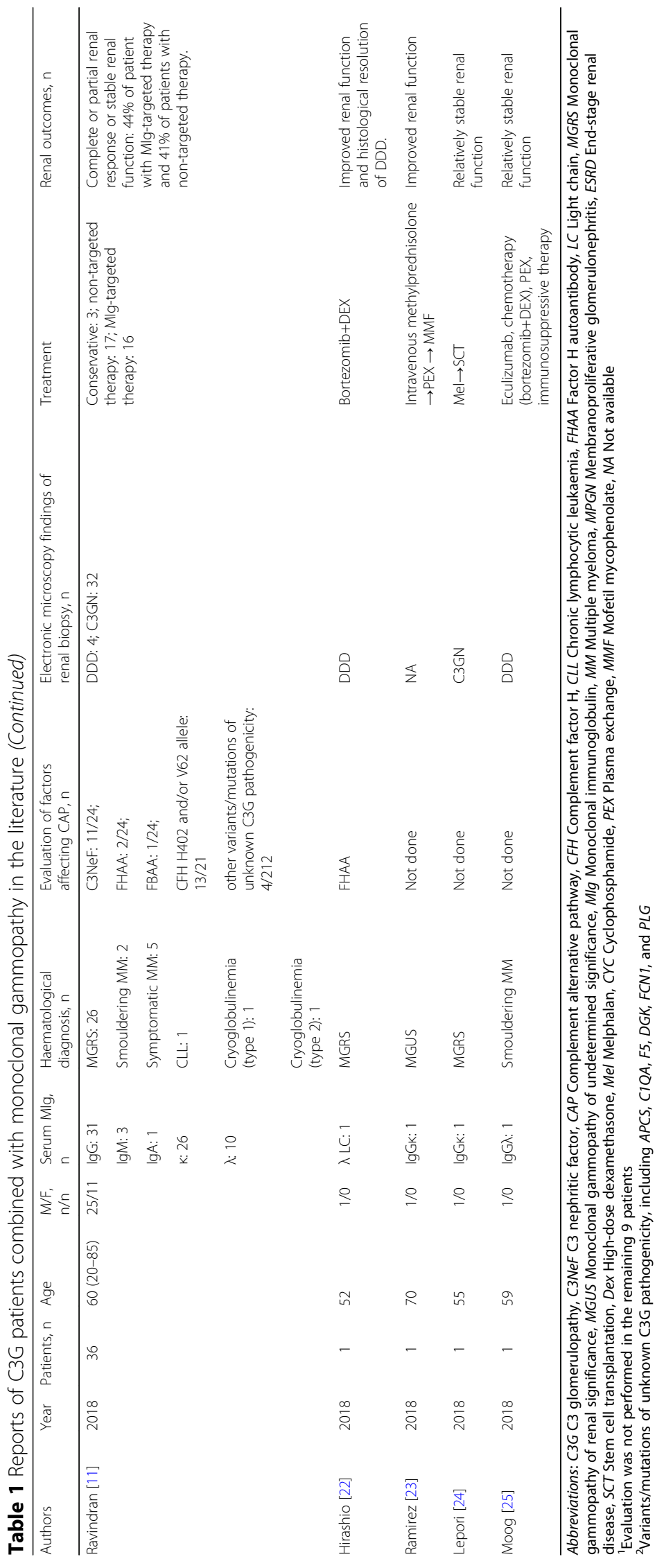


IgG subclasses, which results in its higher molecular mass and the difference in hinge flexibility [27]. The difference in flexibility influences the binding of IgG3 to both $\mathrm{C} 1 \mathrm{q}$ and $\mathrm{Fc} \gamma \mathrm{R}$, and affects the antigen-binding capacity and immune complex formation, which results in a potent pro-inflammation and a particularly effective antibody [26]. The current work aimed to clarify the causal relationship between the MIg and the C3G occurrence, and further explorations of its IgG3 subclass are needed.

In our patient, anti-CFH autoantibody and MIg $(\operatorname{IgG} \lambda)$ were both demonstrated in the serum. In further explorations, we purified the intact and specific IgG against CFH directly and found that the purified antibody was a monoclonal $\operatorname{IgG} \lambda$, which could inhibit the $\mathrm{CFH}$ binding to $\mathrm{C} 3 \mathrm{~b}$ in a dose-dependent manner and accelerate the formation of $\mathrm{C} 3$ convertase $(\mathrm{C} 3 \mathrm{bBb})$ indirectly by interfering with the formation-impeding activity of $\mathrm{CFH}$. Our results highlighted that the MIg-C3G could be attributed to the over-activation of the CAP by the monoclonal anti-CFH IgG $\lambda$. In a previous study, Meri et al. reported that the Ig $\lambda$-chain dimer purified from a patient with membranoproliferative glomerulonephritis served as a mini-antibody directed against CFH SCR3 and was responsible for CAP activation before C3GN was described as a separate entity $[19,20]$, which is consistent with our findings concerning the monoclonal $\operatorname{IgG} \lambda$ of our patient. Importantly, more direct evidences focusing on the effects of the dysregulations of $\mathrm{CFH}$ on the $\mathrm{C} 3$ convertase, could better reflect the uncontrolled CAP activation from our patient.

Interestingly, the $\mathrm{C} 3 \mathrm{NeF}$ activity was also positive in our patient and it turned negative with the disappearance of anti-CFH autoantibodies during disease remission, although the anti-CFH autoantibodies failed to stabilize the C3 convertase directly in our in vitro experiments. It is suggested that the $\mathrm{C} 3 \mathrm{NeF}$, a group of autoantibodies detected in the majority of DDD (86\%) and less (45\%) in C3GN patients [4], could bind to neoepitopes in the newly assembled $\mathrm{C} 3 \mathrm{bBb}$ and increase the half-life of the convertase by stabilizing it against both intrinsic and extrinsic $\mathrm{CFH}$-mediated decay [28, 29]. However, the standard methods of measuring $\mathrm{C} 3 \mathrm{NeF}$ are not currently well established: it is usually identified by residual $\mathrm{Bb}$, haemolysis assays or $\mathrm{C} 3$ breakdown products, and rarely by the direct detection of autoantibodies [28]. We used the C3NeF stabilization ELISA with properdin (COS-P) to identify $\mathrm{C} 3 \mathrm{NeF}$ indirectly here. With further explorations, we found that the anti$\mathrm{CFH}$ autoantibodies could inhibit the $\mathrm{CFH}$ binding to $\mathrm{C} 3 \mathrm{~b}$ and interfered with formation-impeding activity of $\mathrm{CFH}$, thus directly causing the stabilization of $\mathrm{C} 3$ convertase. Thus, we hypothesized that the anti-CFH autoantibodies were distinct from the classical $\mathrm{C} 3 \mathrm{NeF}$, and the detected $\mathrm{C} 3 \mathrm{NeF}$ activity in our study might be due to the effects of the dysregulation of $\mathrm{CFH}$ on the $\mathrm{C} 3$ convertase.

In this case, our patient was successfully treated using immunosuppressive therapy with oral prednisone plus cyclophosphamide although he denied the bortezomib. The treatment for C3G patients with monoclonal gammopathy are mainly based on clinical opinion and experience by now, as there lacked confirmed guidance. In 2013, the study from the Mayo Clinic suggested that if monoclonal gammopathy was due to a MGUS, chemotherapy directly against the pathological clone was preferred [30]. Recent evidences from several observational studies has further supported a clone-directed approach to treat C3G with monoclonal gammopathy $[12,16]$. In the study of Chauvet et al., treatment with clone-directed chemotherapy (29 patients, including bortezomib in 22 patients), compared with treatment without chemotherapy (21 patients, including either immunosuppressive therapy or conservative therapy), was associated with an improved rate of renal response and improved renal survival [12]. Importantly, renal survival was significantly increased among patients achieving a homological response. Data from the Mayo Clinic showed that targeted therapy against the monoclonal gammopathy in 16 patients (five of 16 had MGRS; others had more advanced hematological disease) resulted in a complete or partial renal response or stable renal function in $44 \%$ of patients. Furthermore, 17 patients (all of them had MGRS) received non-targeted therapy (mostly glucocorticoids alone or in combination with other immunosuppressive agents), and non-targeted therapy resulted in a complete or partial renal response in $41 \%$ of patients [16].

Herein, we reported a patient with C3GN who had anti-CFH IgG activity and a monoclonal gammopathy. We further proved that the functional autoantibody was borne by the monoclonal $\operatorname{IgG} \lambda$, and it is the first time that intact monoclonal immunoglobulin could act as an anti-CFH antibody and lead to MGRS-associated C3GN by activating the CAP.

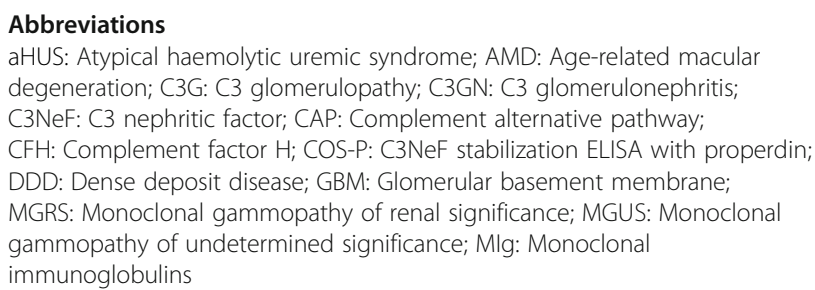

\section{Acknowledgements}

Not applicable.

\section{Authors' contributions}

$\mathrm{L}-\mathrm{LL}$ conducted the experiments and analyzed the data. $\mathrm{L}-\mathrm{ZY}$ and $\mathrm{L}-\mathrm{LL}$ drafted the manuscript. W-SX assisted with the pathological diagnosis, Y-XJ participated in the genetic testing and TY provided suggestions for experiments. Z-MH was involved in study design, assisted with interpretation of data, and provided suggestion for revising the manuscript. FY and YW conceived of the study, participated in the revision of the manuscript, and provided final approval 
of the version of the submitted manuscript. All authors read and approved the manuscript.

\section{Funding}

This work was supported by grants from the National Natural Science Foundation of China to the Innovation Research Group (No. 81621092), the National Natural Science Foundation of China (No. 81670639, No. 81670640 No. 81870477 and No. 81870479) and the Beijing Natural Science Foundation (No. 7172215). These funds are responsible for funding support, guiding the research design and supervising the research process.

\section{Availability of data and materials}

The data used and analyzed during the current study are available from the corresponding author on reasonable request.

\section{Ethics approval and consent to participate}

The study design was approved by the local ethics committee of Peking University First Hospital (No. 20171280) and complied with the Declaration of Helsinki. Written informed consent (No. 1.0) was obtained for blood samples and DNA samples from all individuals.

\section{Consent for publication}

Written informed consent from the reported patient for publication was obtained.

\section{Competing interests}

The authors declare that they have no competing interests.

\section{Author details}

${ }^{1}$ Renal Division, Department of Medicine, Peking University First Hospital, Institute of Nephrology, Peking University, Beijing 100034, People's Republic of China. ${ }^{2}$ Key Laboratory of Renal Disease, Ministry of Health of China, Key Laboratory of CKD Prevention and Treatment, Ministry of Education of China, Beijing 100034, People's Republic of China. ${ }^{3}$ Laboratory of Electron Microscopy, Pathological Centre, Peking University First Hospital, Beijing 100034, People's Republic of China. ${ }^{4}$ Department of Nephrology, Peking University International Hospital, Beijing 102206, People's Republic of China.

${ }^{5}$ Peking-Tsinghua Center for Life Sciences, Beijing, People's Republic of China.

\section{Received: 28 March 2019 Accepted: 22 November 2019}

Published online: 10 December 2019

\section{References}

1. Fakhouri F, Fremeaux-Bacchi V, Noel LH, Cook HT, Pickering MC. C3 glomerulopathy: a new classification. Nat Rev Nephrol. 2010;6(8):494-9.

2. Pickering MC, D'agati VD, Nester CM, Smith RJ, Haas M, Appel GB, et al. C3 glomerulopathy: consensus report. Kidney Int. 2013;84(6):1079-89.

3. Zhang Y, Meyer NC, Wang K, Nishimura C, Frees K, Jones M, et al. Causes of alternative pathway dysregulation in dense deposit disease. Clin J Am Soc Nephrol. 2012;7(2):265-74.

4. Servais A, Noel LH, Roumenina LT, Le Quintrec M, Ngo S, Dragon-Durey MA et al. Acquired and genetic complement abnormalities play a critical role in dense deposit disease and other C3 glomerulopathies. Kidney Int. 2012; 82(4):454-64.

5. Lloyd IE, Gallan A, Huston HK, Raphael KL, Miller DV, Revelo MP, et al. C3 glomerulopathy in adults: a distinct patient subset showing frequent association with monoclonal gammopathy and poor renal outcome. Clin Kidney J. 2016;9(6):794-9.

6. Bridoux F, Leung N, Hutchison CA, Touchard G, Sethi S, Fermand JP, et al. Diagnosis of monoclonal gammopathy of renal significance. Kidney Int. 2015;87(4):698-711.

7. Leung N, Bridoux F, Batuman V, Chaidos A, Cockwell P, D'agati VD, et al. The evaluation of monoclonal gammopathy of renal significance: a consensus report of the international kidney and monoclonal Gammopathy research group. Nat Rev Nephrol. 2019;15(1):45-59.

8. Bridoux F, Desport E, Fremeaux-Bacchi V, Chong CF, Gombert JM, Lacombe C, et al. Glomerulonephritis with isolated C3 deposits and monoclonal gammopathy: a fortuitous association? Clin J Am Soc Nephrol. 2011;6(9): 2165-74.
9. Sethi S, Sukov WR, Zhang Y, Fervenza FC, Lager DJ, Miller DV, et al. Dense deposit disease associated with monoclonal gammopathy of undetermined significance. Am J Kidney Dis. 2010;56(5):977-82.

10. Zand L, Kattah A, Fervenza FC, Smith RJ, Nasr SH, Zhang Y, et al. C3 glomerulonephritis associated with monoclonal gammopathy: a case series. Am J Kidney Dis. 2013;62(3):506-14.

11. Ravindran A, Fervenza FC, Smith RJH, De Vriese AS, Sethi S. C3 Glomerulopathy: ten Years' experience at Mayo Clinic. Mayo Clin Proc. 2018; 93(8):991-1008.

12. Chauvet S, Fremeaux-Bacchi V, Petitprez F, Karras A, Daniel L, Burtey S, et al. Treatment of B-cell disorder improves renal outcome of patients with monoclonal gammopathy-associated C3 glomerulopathy. Blood. 2017; 129(11):1437-47.

13. Chauvet $S$, Roumenina LT, Aucouturier P, Marinozzi MC, Dragon-Durey MA, Karras A, et al. Both monoclonal and polyclonal immunoglobulin contingents mediate complement activation in monoclonal Gammopathy associated-C3 Glomerulopathy. Front Immunol. 2018;9:2260.

14. Daccueil F, Masani NN, D'agati VD, Shirazian S. A 61-year-old man with Membranoproliferative glomerulonephritis. Am J Kidney Dis. 2017;70(6):A16-20.

15. Timmermans $S$, Van Paassen $P$, Limburg RR. Conservative treatment for C3 glomerulopathy and monoclonal Ig. Kidney Int. 2018;94(3):632.

16. Ravindran A, Fervenza FC, Smith RJH, Sethi S. C3 glomerulopathy associated with monoclonal Ig is a distinct subtype. Kidney Int. 2018;94(1):178-86.

17. Yin G, Cheng Z, Zeng CH, Liu ZH. C3 glomerulonephritis in multiple myeloma: a case report and literature review. Medicine (Baltimore). 2016; 95(37):e4843.

18. Kyle Ra TT, Rajkumar SV, Larson DR, Plevak MF, Offord JR, Dispenzieri A, Katzmann JA, Melton $\sqcup$ 3rd. Prevalence of monoclonal Gammopathy of undetermined significance. N Engl J Med. 2006;354(13):1362-9.

19. Meri S, Koistinen V, Miettinen A, Tornroth T, Seppala IJ. Activation of the alternative pathway of complement by monoclonal lambda light chains in membranoproliferative glomerulonephritis. J Exp Med. 1992;175(4):939-50

20. Jokiranta TS, Solomon A, Pangburn MK, Zipfel PF, Meri S. Nephritogenic lambda light chain dimer: a unique human miniautoantibody against complement factor H. J Immunol. 1999:163(8):4590-6.

21. Hamzi MA, Zniber A, Badaoui GE, Mahtat E, Alhamany Z, Bayahia R, et al. C3 glomerulopathy associated to multiple myeloma successfully treated by autologous stem cell transplant. Indian J Nephrol. 2017;27(2):141-4.

22. Hirashio S, Satoh A, Arima T, Mandai K, Awaya T, Oshima K, et al. Favorable effect of bortezomib in dense deposit disease associated with monoclonal gammopathy: a case report. BMC Nephrol. 2018;19(1):108.

23. Ramirez GA, De Luca G, Dell'antonio G, Baldissera E, Suma GV, Tresoldi M, et al. C3-glomerulopathy and MGUS: the skin beyond the kidney. QJM. 2018:111(3):187-8

24. Lepori N, Cheungpasitporn W, Sethi S, Murray D, Kumar S, Leung N, et al. High-dose melphalan and autologous hematopoietic stem cell transplant in patient with C3 glomerulonephritis associated with monoclonal gammopathy. Clin Nephrol. 2018;89(4):291-9.

25. Moog P, Jost PJ, Buttner-Herold M. Eculizumab as salvage therapy for recurrent monoclonal gammopathy-induced C3 glomerulopathy in a kidney allograft. BMC Nephrol. 2018;19(1):106.

26. Gestur Vidarsson GD, Rispens T. IgG subclasses and allotypes- from structure to effector functions. Front Immunol. 2014:5.520

27. Roux Kh SL, Michaelsen TE. Flexibility of human IgG subclasses. J Immunol. 1997:159(7):3372-82.

28. Paixao-Cavalcante D, Lopez-Trascasa M, Skattum L, Giclas PC, Goodship TH, De Cordoba SR, et al. Sensitive and specific assays for C3 nephritic factors clarify mechanisms underlying complement dysregulation. Kidney Int. 2012; 82(10):1084-92.

29. Donadelli R, Pulieri P, Piras R, latropoulos P, Valoti E, Benigni A, et al. Unraveling the molecular mechanisms underlying complement Dysregulation by nephritic factors in C3G and IC-MPGN. Front Immunol. 2018;9:2329.

30. Sethi S, Rajkumar SV. Monoclonal gammopathy-associated proliferative glomerulonephritis. Mayo Clin Proc. 2013;88(11):1284-93.

\section{Publisher's Note}

Springer Nature remains neutral with regard to jurisdictional claims in published maps and institutional affiliations. 